PROCEEDINGS OF THE

AMERICAN MATHEMATICAL SOCIETY

Volume 126, Number 4, April 1998, Pages 965-971

S 0002-9939(98)04363-9

\title{
REDUCIBILITY OF TRANSLATES OF DICKSON POLYNOMIALS
}

\author{
GERHARD TURNWALD
}

(Communicated by William W. Adams)

\begin{abstract}
Let $K$ be a field and $a, b \in K$. The Dickson polynomial $D_{n}(x, a)$ is characterized by the equation $D_{n}(x+(a / x), a)=x^{n}+(a / x)^{n}$. We prove that $D_{n}(x, a)+b \in K[x]$ is reducible if and only if there is a prime $p \mid n$ such that $b=-D_{p}\left(c, a^{n / p}\right)$ for some $c \in K$, or $n=4 k$ and $b=4 c^{4}-8 a^{k} c^{2}+2 a^{2 k}$ for some $c \in K$. This result generalizes the well-known reducibility criterion for binomials; and it provides a reducibility criterion for $T_{n}(x)+c$ where $T_{n}(x)$ denotes the Chebyshev polynomial of degree $n$.
\end{abstract}

\section{INTRODUCTION}

Let $K$ be a field. For every positive integer $n$ and every $a \in K$ there is a unique polynomial $D_{n}(x, a) \in K[x]$ such that $D_{n}(x+(a / x), a)=x^{n}+(a / x)^{n}$. This polynomial is monic of degree $n$ and the coefficients are polynomials in $a$ independent of $K$ (see 1.1). It is called the Dickson polynomial of degree $n$ with parameter $a$. Note that $D_{n}(x, 0)=x^{n}$. The following theorem is the main result of the present paper. (See 2.5; cf. 2.4 and 2.6.)

Theorem. The polynomial $D_{n}(x, a)+b \in K[x]$ is reducible iff there is a prime $p \mid n$ such that $b=-D_{p}\left(c, a^{n / p}\right)$ for some $c \in K$, or $n=4 k$ and $b=4 c^{4}-8 a^{k} c^{2}+2 a^{2 k}$.

In the case $a=0$ the above theorem yields the well-known description of reducible binomials (see 3.1). For finite fields (and $a \neq 0$ ) a reducibility criterion in terms of the order (in the multiplicative group of $K$ ) of a zero of $x^{2}+b x+a^{n}$ was recently found by Gao and Mullen. In the final section we show how to deduce their theorem from our results. The final section also contains a very simple condition for the reducibility of $D_{n}(x, a)$. It turns out that the answer does not depend on $a$, provided that $a \neq 0$ and $n>2$.

The Chebyshev polynomial $T_{n}(x)$ (usually defined by the property $T_{n}(\cos \varphi)=$ $\cos n \varphi)$ has integral coefficients and satisfies $T_{n}\left(\left(x+x^{-1}\right) / 2\right)=\left(x^{n}+x^{-n}\right) / 2$. Hence $T_{n}(x)$ may be defined over every field $K$; and $T_{n}(x)=D_{n}(2 x, 1) / 2$ if $\operatorname{char} K \neq 2$. Thus, if $c \in K$ and char $K \neq 2, T_{n}(x)+c$ is reducible over $K$ iff $D_{n}(x, 1)+2 c$ is reducible. In this situation the above theorem implies that $T_{n}(x)+c$ is reducible iff there is a prime $p \mid n$ such that $c=-T_{p}(d)$ for some $d \in K$, or $4 \mid n$ and $c=$ $2 d^{4}-4 d^{2}+1$ for some $d \in K$.

Received by the editors September 10, 1996.

1991 Mathematics Subject Classification. Primary 12E10; Secondary 11T06.

Key words and phrases. Dickson polynomials, Chebyshev polynomials, binomials, reducibility.

(C)1998 American Mathematical Society 
The notation $K$ will be reserved for fields. The multiplicative group and the characteristic of $K$ are denoted by $K^{\times}$and char $K$, respectively; and $\bar{K}$ denotes an algebraic closure of $K$. We always tacitly assume that $k, m, n$ denote positive integers. For every $m$ we write $K^{(m)}=\left\{a^{m}: a \in K\right\}$. For an extension field $L$ of $K$ of finite degree $[L: K]$ we let $\mathrm{N}_{L / K}$ denote the norm map from $L$ into $K$. The greatest common divisor of $k$ and $m$ will be denoted by $(k, m)$. An expression like $\gamma+a / \gamma$ is to be understood as $\gamma+(a / \gamma)$. We abbreviate 'if and only if' by 'iff'.

\section{Preliminaries}

1.1 Remark. The main theorem on symmetric polynomials shows that for every $n$ there is a unique polynomial $D_{n}(x, y) \in \mathbf{Z}[x, y]$ such that $D_{n}(x+y, x y)=x^{n}+y^{n}$. Let $K$ be a field and $a \in K$. Then $D_{n}(x, a) \in K[x]$ is characterized by the property $D_{n}(x+a / x, a)=x^{n}+(a / x)^{n}$. Moreover, it is clear that $D_{n}(x, a)$ is monic and has degree $n$. It follows that the Dickson polynomials (as described in the introduction) exist.

The defining property yields $D_{1}(x, a)=x, D_{2}(x, a)=x^{2}-2 a$, and $D_{n}(x, a)=$ $x D_{n-1}(x, a)-a D_{n-2}(x, a)$ if $n \geq 3$. Conversely, the Dickson polynomials can be defined recursively by this relation. One can prove that

$$
D_{n}(x, a)=\sum_{k \leq n / 2} \frac{n}{n-k}\left(\begin{array}{c}
n-k \\
k
\end{array}\right)(-a)^{k} x^{n-2 k} .
$$

This explicit formula is irrelevant for our purposes, however. (See [4] for more information on Dickson polynomials.)

1.2 Lemma. The following properties hold for all $a \in K$ and all $n$.

(a) $D_{2}(x, a)=x^{2}-2 a, D_{4}(x, a)=x^{4}-4 a x^{2}+2 a^{2}$.

(b) $D_{n}(b+c, a)=b^{n}+c^{n}$ for all $b, c \in K$ with $b c=a$.

(c) If $n$ is odd (even) then $D_{n}(x, a)$ only contains odd (even) powers of $x$.

(d) $D_{k m}(x, a)=D_{m}\left(D_{k}(x, a), a^{k}\right)$ for all $k, m$.

Proof. Parts (a) and (c) are clear from the recurrence relation in 1.1, (b) follows from the definition, and (d) holds since both sides yield $x^{k m}+(a / x)^{k m}$ when $x$ is replaced by $x+a / x$.

1.3 Lemma. Let $a, b \in K$.

(a) Let $\alpha \in \bar{K}$ be a zero of $D_{m}\left(x, a^{k}\right)+b$. Then $D_{k m}(x, a)+b$ is reducible over $K$ iff $D_{m}\left(x, a^{k}\right)+b$ is reducible over $K$ or $D_{k}(x, a)-\alpha$ is reducible over $K(\alpha)$.

(b) Let $m>1$. Assume that $D_{2 m}(x, a)+b$ is reducible over $K$ and $D_{m}\left(x, a^{2}\right)+b$ is irreducible. Then $2 a^{m}+(-1)^{m} b \in K^{(2)} \backslash\{0\}$.

Proof. (a) Let $\xi \in \bar{K}$ be a zero of $D_{k}(x, a)-\alpha$. From $1.2(\mathrm{~d})$ we see that $D_{k m}(\xi, a)+$ $b=0$. Hence $D_{k m}(x, a)+b$ is reducible iff $[K(\xi): K]<k m$. The assertion easily follows by making use of the equality $[K(\xi): K]=[K(\xi): K(\alpha)][K(\alpha): K]$.

(b) Let $\alpha \in \bar{K}$ be a zero of $D_{m}\left(x, a^{2}\right)+b$ and let $L=K(\alpha)$. From (a) we see that $D_{2}(x, a)-\alpha$ is reducible over $L$, i.e., $2 a+\alpha \in L^{(2)}$. Thus $\mathrm{N}_{L / K}(2 a+\alpha) \in K^{(2)}$. Note that $2 a+\alpha$ is a zero of the irreducible polynomial $f(x)=D_{m}\left(x-2 a, a^{2}\right)+b$. Hence $\mathrm{N}_{L / K}(2 a+\alpha)=(-1)^{m} f(0) \neq 0$. We have $D_{m}\left(-2 a, a^{2}\right)=2(-a)^{m}$ (by $\left.1.2(\mathrm{~b})\right)$ and hence $f(0)=2(-a)^{m}+b$. Thus $2 a^{m}+(-1)^{m} b=\mathrm{N}_{L / K}(2 a+\alpha) \in K^{(2)} \backslash\{0\}$. 
1.4 Lemma. Let char $K \neq 2, a, b \in K$, and $f(x)=x^{2}+a x+b$. Then $f\left(x^{2}\right)$ is reducible over $K$ iff there exists $c \in K$ such that (i) $4 b=a^{2}-c^{2}$ or (ii) $4 b=\left(a+c^{2}\right)^{2}$.

In case (i) we have $f\left(x^{2}\right)=\left(x^{2}+(a-c) / 2\right)\left(x^{2}+(a+c) / 2\right)$ and in case (ii) we have $f\left(x^{2}\right)=\left(x^{2}+c x+\left(a+c^{2}\right) / 2\right)\left(x^{2}-c x+\left(a+c^{2}\right) / 2\right)$.

Proof. Assume that $f\left(x^{2}\right)$ is reducible. If $f(x)$ is reducible then $a^{2}-4 b=c^{2}$ with $c \in K$. In the sequel we assume that $f(x)$ is irreducible. For every zero $\alpha \in \bar{K}$ of $f\left(x^{2}\right)$ we have $[K(\alpha): K]=2$ since $\left[K\left(\alpha^{2}\right): K\right]=2$ and $[K(\alpha): K]<4$. Hence $f\left(x^{2}\right)=g(x) h(x)$ where $g(x), h(x) \in K[x]$ are monic irreducible polynomials of degree 2. Let $g(x)=x^{2}+c x+d$. If $g(x)=g(-x)$ then $c=0$ and $-d$ is a zero of $f(x)$, a contradiction. Since $g(-x)$ is a monic irreducible factor of $f\left(x^{2}\right)$, we obtain $h(x)=g(-x)$ and thus $f\left(x^{2}\right)=x^{4}+\left(2 d-c^{2}\right) x^{2}+d^{2}$. Hence $4 b=(2 d)^{2}=\left(a+c^{2}\right)^{2}$. The converse is clear.

1.5 Lemma. Let $a, b \in K$.

(a) Let $D_{4}(x, a)+b$ be reducible. Then $D_{2}\left(x, a^{2}\right)+b$ is reducible or $b=4 c^{4}-$ $8 a c^{2}+2 a^{2}$ for some $c \in K$.

(b) Suppose that $b=4 c^{4}-8 a c^{2}+2 a^{2}$ for some $c \in K$. Then $D_{4}(x, a)+b=$ $\left(x^{2}-2 c x+2\left(c^{2}-a\right)\right)\left(x^{2}+2 c x+2\left(c^{2}-a\right)\right)$ is reducible and $2 a^{2}+b \in K^{(2)}$; and $D_{2}\left(x, a^{2}\right)+b$ is irreducible iff $c \in K^{\times}$and $2 a-c^{2} \notin K^{(2)}$.

Proof. (a) Suppose that $D_{2}\left(x, a^{2}\right)+b$ is irreducible, i.e., $2 a^{2}-b \notin K^{(2)}$. It follows from $1.3(\mathrm{~b})$ that $\operatorname{char} K \neq 2$. Recall that $D_{4}(x, a)=x^{4}-4 a x^{2}+2 a^{2}$ (by $1.2(\mathrm{a})$ ). The assertion is obtained by applying 1.4 to $f(x)=x^{2}-4 a x+2 a^{2}+b$.

(b) The first part holds since $2 a^{2}+b=4\left(c^{2}-a\right)^{2}$ and $D_{4}(x, a)+b=x^{4}-$ $4 a x^{2}+4\left(c^{2}-a\right)^{2}$. The second part follows from $2 a^{2}-b=4 c^{2}\left(2 a-c^{2}\right)$. (Note that $2 a-c^{2} \notin K^{(2)}$ implies that $\operatorname{char} K \neq 2$.)

\section{The MAIN RESUlts}

2.1 Proposition. Let $a \in K^{\times}, b \in K$, and $n>2$. Let $\beta \in \bar{K}$ be a zero of $x^{2}+b x+a^{n}$.

(a) Let $n$ be odd. Then $D_{n}(x, a)+b$ is reducible over $K$ iff $x^{n}-\beta$ is reducible over $K(\beta)$.

(b) Assume that $\beta \in K$. Then $D_{n}(x, a)+b$ is reducible iff $x^{n}-\beta$ is reducible or $b^{2}=4 a^{n}$.

Proof. Let $\gamma \in \bar{K}$ be a zero of $x^{n}-\beta, \alpha=\gamma+a / \gamma$, and $L=K(\alpha)$. Note that $D_{n}(\alpha, a)=\beta+a^{n} / \beta=-b$. Hence $D_{n}(x, a)+b$ is reducible iff $[L: K] \neq n$.

(a) Let $n_{1}=[K(\gamma): K(\beta)]$ and $n_{2}=[L: K]$. It remains to prove that $n_{1} \neq n$ iff $n_{2} \neq n$. We have $[K(\gamma): K]=n_{1} m_{1}$ with $m_{1}=[K(\beta): K] \leq 2$, and $[K(\gamma): K]=m_{2} n_{2}$ with $m_{2}=[K(\gamma): L] \leq 2$. The assertion follows since $n_{1}, n_{2} \leq n$ and $n$ is odd.

(b) Note that $b^{2}=4 a^{n}$ holds iff $\beta=a^{n} / \beta ; x^{n}-\beta$ is reducible iff $[K(\gamma): K] \neq n$. We have $L \subseteq K(\gamma)$ and $\gamma$ is a zero of $f(x)=(x-\gamma)(x-a / \gamma) \in L[x]$. If $\beta \neq a^{n} / \beta$ then $\gamma$ and $a / \gamma$ are not conjugate over $L$. In this case it follows that $\gamma \in L$ and $L=K(\gamma)$; and thus the assertion holds. Now suppose that $\beta=a^{n} / \beta$. Assume that $[L: K]=n$. Then it follows that $L=K(\gamma), \gamma \neq a / \gamma($ since $n>2)$, and $x^{n}-\beta$ is irreducible. Since $a / \gamma$ is a zero of $x^{n}-\beta$, there exists a $K$-embedding $\sigma: K(\gamma) \rightarrow \bar{K}$ with $\sigma(\gamma)=a / \gamma$. Then $\sigma(\alpha)=\alpha$ and thus $\sigma$ is trivial on $L$. Hence $L \neq K(\gamma)$ since $\sigma(\gamma) \neq \gamma$. This contradiction proves that $[L: K] \neq n$. The assertion follows. 
2.2 Proposition. Let $a, b \in K$. Let $\beta \in \bar{K}$ be a zero of $x^{2}+b x+a^{4}$. Assume that $L=K(\beta) \neq K$ and $D_{2}\left(x, a^{2}\right)+b$ is irreducible.

(a) $D_{4}(x, a)+b$ is reducible iff there exists $\eta \in L$ such that $\beta=-4 \eta^{4}$ and $2 \mathrm{~N}_{L / K}(\eta)=a$.

(b) Suppose that $\zeta^{2}=-1, \beta=-4 \eta^{4}$, and $2 a^{2}+b=\varepsilon c^{2}$ with $\zeta, \eta \in L, \varepsilon= \pm 1$, and $c \in K$. Then $D_{4}(x, a)+b$ is reducible, $\zeta \in K$, and $-4 \in K^{(4)}$.

Proof. (a) Suppose that $D_{4}(x, a)+b$ is reducible. By 1.5 there is $c \in K^{\times}$such that $b=4 c^{4}-8 a c^{2}+2 a^{2}$ and $2 a-c^{2} \notin K^{(2)}$. It follows that $\operatorname{char} K \neq 2$ and $c^{2} \neq a$. Let $\xi=\left(\beta-a^{2}\right) /\left(a-c^{2}\right)$. Then $\xi \in L, \xi^{2}=-4 \beta$, and $\xi=(2 a+\xi)^{2} / 4 c^{2}$. Hence $\beta=-4 \eta^{4}$ with $\eta=(2 a+\xi) / 4 c$. From $\xi^{2}+4\left(a-c^{2}\right) \xi+4 a^{2}=0$ we obtain $\eta^{2}-c \eta+a / 2=0 ;$ and thus $\mathrm{N}_{L / K}(\eta)=a / 2$.

Conversely, suppose that $\beta=-4 \eta^{4}$ and $\mathrm{N}_{L / K}(\eta)=a / 2$. Let $\sigma$ denote the nontrivial $K$-automorphism of $L$. Then $c=\eta+\sigma(\eta) \in K, \eta \sigma(\eta)=a / 2$, and $-b=\beta+\sigma(\beta)$. Hence $b / 4=\eta^{4}+\sigma(\eta)^{4}=D_{4}(c, a / 2)=c^{4}-2 a c^{2}+a^{2} / 2$ and $D_{4}(x, a)+b$ is reducible according to $1.5(\mathrm{~b})$.

(b) Note that $c \neq 0$ since otherwise $\beta=a^{2} \in K$. Let $\xi=\left(\beta-a^{2}\right) / c$. Then $\xi^{2}=-\varepsilon \beta$ and $\xi^{2}+\varepsilon c \xi+\varepsilon a^{2}=0$. We have $-4=(2 \zeta)^{2}=(1+\zeta)^{4}$ and hence $\beta \in L^{(4)}$. Suppose that $\varepsilon=-1$. Then $\xi^{2} \in L^{(4)}$ and hence $\xi \in L^{(2)}$ (since $\left.-1 \in L^{(2)}\right)$. Thus $-a^{2}=\mathrm{N}_{L / K}(\xi) \in K^{(2)}$ and $-1 \in K^{(2)}$. Hence without loss of generality we may restrict to the case $\varepsilon=1$. Note that $\operatorname{char} K \neq 2($ since $\beta \neq 0)$.

Assume that $\zeta \notin K$. Then there are $\lambda, \mu \in K$ such that $(\lambda+\mu \beta)^{2}=-1$ and $\mu \neq 0$. It follows that $\beta^{2}+2 \lambda \mu^{-1} \beta+\left(\lambda^{2}+1\right) / \mu^{2}=0$. Hence $2 \lambda / \mu=b$, $\left(\lambda^{2}+1\right) / \mu^{2}=a^{4}$, and $4 a^{4}-b^{2}=4 / \mu^{2} \in K^{(2)} \backslash\{0\}$. We obtain $2 a^{2}-b \in K^{(2)}$ since $2 a^{2}+b \in K^{(2)}$. This contradicts the irreducibility of $D_{2}\left(x, a^{2}\right)+b$. Thus we conclude that $\zeta \in K$.

From $\xi^{2}=-\beta=4 \eta^{4}$ we get $\xi= \pm 2 \eta^{2}$. Hence $a^{2}=\mathrm{N}_{L / K}(\xi)=4 \mathrm{~N}_{L / K}(\eta)^{2}$. It follows that $\mathrm{N}_{L / K}(\eta)= \pm a / 2$. Note that $\mathrm{N}_{L / K}(\zeta)=\zeta^{2}$ and $\mathrm{N}_{L / K}(\zeta \eta)=$ $-\mathrm{N}_{L / K}(\eta)$. Possibly replacing $\eta$ by $\zeta \eta$, we may thus assume that $\beta=-4 \eta^{4}$ and $\mathrm{N}_{L / K}(\eta)=a / 2$. Then $D_{4}(x, a)+b$ is reducible according to (a) and the proof is finished.

We will use the following part (for odd degree) of the well-known reducibility criterion for binomials (see 3.1). A proof may be found in [3, p.297], [5, p.662], and [7, p.91].

2.3 Lemma. Let $n$ be odd and $a \in K$. Then $x^{n}-a$ is reducible (over $K$ ) iff for some prime $p \mid n$ we have $a \in K^{(p)}$.

2.4 Theorem. Let $a, b \in K$. If $D_{n}(x, a)+b$ is irreducible then $D_{m}\left(x, a^{n / m}\right)+b$ is irreducible for every $m \mid n$. If $D_{n}(x, a)+b$ is reducible over $K$ then $D_{p}\left(x, a^{n / p}\right)+b$ is reducible for some prime $p \mid n$, or $n=4 k$ and $D_{4}\left(x, a^{k}\right)+b$ is reducible.

Proof. The first part holds by 1.3(a). By induction we may assume that the second part is valid (for all $K$ and all $a, b \in K$ ) if $n$ is replaced by $1, \ldots, n-1$. Suppose that $D_{n}(x, a)+b$ is reducible. First we assume that $n$ is odd. If $a=0$ then the assertion follows from 2.3 since $D_{n}(x, 0)=x^{n}$. If $a \neq 0$ then, according to 2.1(a), $x^{n}-\beta$ is reducible over $K(\beta)$ and we have to show that $x^{p}-\beta$ is reducible over $K(\beta)$ for some prime $p \mid n$. Again, the assertion follows by making use of 2.3 .

In the sequel we suppose that $n=2 m$. The proof is finished if $D_{2}\left(x, a^{m}\right)+b$ is reducible. Thus we assume that $D_{2}\left(x, a^{m}\right)+b$ is irreducible. Let $\gamma \in \bar{K}$ be a zero 
of $D_{2}\left(x, a^{m}\right)+b$ (i.e., $\gamma^{2}=2 a^{m}-b$ ). From $1.3\left(\right.$ a) we obtain that $D_{m}(x, a)-\gamma$ is reducible over $L=K(\gamma)$. By induction, $D_{p}\left(x, a^{m / p}\right)-\gamma$ is reducible over $L$ for some prime $p \mid m$, or $4 \mid m$ and $D_{4}\left(x, a^{m / 4}\right)-\gamma$ is reducible over $L$.

If $2 \mid m$ and $D_{2}\left(x, a^{m / 2}\right)-\gamma$ is reducible then there are $\lambda, \mu \in K$ such that $2 a^{m / 2}+\gamma=(\lambda+\mu \gamma)^{2}$. We obtain $2 a^{m / 2}=\lambda^{2}+\left(2 a^{m}-b\right) \mu^{2}$ and $1=2 \lambda \mu$. Thus $b=\left(\lambda^{2}-2 a^{m / 2}\right) \mu^{-2}+2 a^{m}=4 \lambda^{4}-8 a^{m / 2} \lambda^{2}+2 a^{m}$ and $1.5(\mathrm{~b})$ implies that $D_{4}\left(x, a^{n / 4}\right)+b$ is reducible.

Now suppose that $p$ is an odd prime such that $p \mid m$ and $D_{p}\left(x, a^{m / p}\right)-\gamma$ is reducible over $L$. According to $1.3(\mathrm{a}), D_{2 p}\left(x, a^{m / p}\right)+b$ is reducible over $K$. Hence $1.3(\mathrm{~b})$ implies that $D_{p}\left(x, a^{n / p}\right)+b$ is reducible (since $2 a^{m}-b \notin K^{(2)}$ ).

Finally we suppose that $4 \mid m, D_{4}\left(x, a^{m / 4}\right)-\gamma$ is reducible, and $D_{2}\left(x, a^{m / 2}\right)-\gamma$ is irreducible over $L$. It follows from $1.3(\mathrm{~b})$ that $2 a^{m / 2}-\gamma \in L^{(2)} \backslash\{0\}$. Note that $2 a^{m / 2}+\gamma \notin L^{(2)}$ (since $D_{2}\left(x, a^{m / 2}\right)-\gamma$ is irreducible). Hence $2 a^{m}+b=4 a^{m}-\gamma^{2} \notin$ $L^{(2)}$. Thus $1.3(\mathrm{~b})$ yields that $D_{m}\left(x, a^{2}\right)+b$ is reducible and our assertion follows by induction.

2.5 Theorem. Let $a, b \in K$ and $n$ be a positive integer. Then $D_{n}(x, a)+b \in K[x]$ is reducible iff there is a prime $p \mid n$ such that $b=-D_{p}\left(c, a^{n / p}\right)$ for some $c \in K$, or $n=4 k$ and $b=4 c^{4}-8 a^{k} c^{2}+2 a^{2 k}$ for some $c \in K$.

Proof. According to 2.4 and 1.5, we may restrict to the case where $n=p$ is a prime. We have to prove that the reducibility of $D_{p}(x, a)+b$ implies that there is a zero in $K$. The assertion is evident if $p=2$. Thus we assume that $p$ is odd. If $a=0$ then 2.3 applies (since $D_{p}(x, 0)=x^{p}$ ). In the sequel we suppose $a \neq 0$. Choose $\beta \in \bar{K}$ such that $\beta^{2}+b \beta+a^{p}=0$. It follows from 2.1(a) that $x^{p}-\beta$ is reducible over $L=K(\beta)$. Hence (by 2.3) $\beta=\gamma^{p}$ for suitable $\gamma \in L$. From $D_{p}(\gamma+a / \gamma, a)=\beta+a^{p} / \beta=-b$ we see that it is sufficient to show that $\gamma+a / \gamma \in K$ for suitably chosen $\gamma$.

If $L=K$ then we are done. Hence we assume $L \neq K$. Then $[L: K]=2$ and $\mathrm{N}_{L / K}(\beta)=a^{p}$. From $\mathrm{N}_{L / K}(\gamma)^{p}=\mathrm{N}_{L / K}(\beta)$ we get $\mathrm{N}_{L / K}(\gamma)=\zeta a$ where $\zeta \in K$ and $\zeta^{p}=1$. Let $c=\zeta^{-(p+1) / 2}$. Then $\mathrm{N}_{L / K}(c)=c^{2}=\zeta^{-1}$ and $\mathrm{N}_{L / K}(c \gamma)=a$. Hence replacing $\gamma$ by $c \gamma$ we may assume $\mathrm{N}_{L / K}(\gamma)=a$ and then it follows that $\gamma+a / \gamma \in K$

2.6 Remark. (a) The condition $b=4 c^{4}-8 a^{k} c^{2}+2 a^{2 k}$ may be expressed in the form $b=D_{4}\left(\sqrt{2} c, a^{k}\right)$. In this case $D_{4}\left(x, a^{k}\right)+b$ is the product of $D_{2}\left(x-c, a^{k}\right)+c^{2}$ and $D_{2}\left(x+c, a^{k}\right)+c^{2}$ (cf. 1.5(b)).

(b) If $b=4 c^{4}-8 a^{k} c^{2}+2 a^{2 k}$, then $2 a^{2 k}+b=4\left(a^{k}-c^{2}\right)^{2}$ and $2 a^{2 k}-b=$ $4 c^{2}\left(2 a^{k}-c^{2}\right)$. If $D_{2}\left(x, a^{2 k}\right)+b$ is irreducible then $2 a^{2 k}-b \notin K^{(2)}$ and hence char $K \neq 2, c \neq 0,2 a^{k}-c^{2} \notin K^{(2)}$, and $2 a^{2 k}+b \neq 0$ (since otherwise $2 a^{k}-c^{2}=c^{2}$ ). Thus these conditions may be added in the formulation of Theorem 2.5.

(c) The condition $b=4 c^{4}-8 a^{k} c^{2}+2 a^{2 k}$ holds iff there is a square root $d \in K$ of $2 a^{2 k}+b$ such that $a^{k}+d / 2 \in K^{(2)}$.

(d) Theorem 2.4 follows from 2.5 .

\section{Special CASES}

The following result is well-known. It is sometimes called the Vahlen-Capelli theorem. For odd $n$ it was quoted as Lemma 2.3.

3.1 Theorem. Let $a \in K$. Then $x^{n}-a$ is reducible over $K$ iff for some prime $p \mid n$ we have $a \in K^{(p)}$, or $4 \mid n$ and $a \in-4 K^{(4)}$. 
Proof. This follows from 2.5 since $D_{n}(x, 0)=x^{n}$.

3.2 Theorem. Let $n>2$ and $a \in K^{\times}$. Then $D_{n}(x, a)$ is irreducible iff $n$ is a power of 2 and $2 \notin K^{(2)}$.

Proof. Suppose $D_{n}(x, a)$ is irreducible. If $m$ is odd then (by 1.2(c)) $D_{m}\left(x, a^{n / m}\right)$ is divisible by $x$. Hence 1.3(a) (or 2.5) shows that $n$ is a power of 2 and $D_{2}\left(x, a^{n / 2}\right.$ ) is irreducible, i.e., $2 a^{n / 2} \notin K^{(2)}$. Thus $2 \notin K^{(2)}$. Conversely, suppose that $n$ is a power of 2 and $2 \notin K^{(2)}$. Then $2 a^{n / 2} \notin K^{(2)}$ and hence 1.3(b) (or 2.5) implies that $D_{4}\left(x, a^{n / 4}\right)$ is irreducible. From 2.5 we conclude that $D_{n}(x, a)$ is irreducible.

3.3 Remark. In the case $|K|<\infty$ the factorization of $D_{n}(x, a)$ has been found recently by Chou [1]. In the case where $K$ is the field of rational numbers the factorization of Chebyshev polynomials is known (see [6, p.228]).

3.4 Lemma. Let $|K|<\infty$ and $a, b \in K$. Let $\beta \in \bar{K}$ be a zero of $x^{2}+b x+a^{4}$. Assume that $L=K(\beta) \neq K$. Then $D_{4}(x, a)+b$ is reducible iff $2 a^{2}-b \in K^{(2)}$ or $\beta \in L^{(4)}$.

Proof. If $q$ is even then $D_{4}(x, a)+b=x^{4}+b$ is reducible and $L^{(4)}=L$. In the sequel we assume that $q$ is odd. Note that $-1 \in L^{(2)}$. If $\zeta^{2}=-1$ then $(1+\zeta)^{4}=-4$. Hence $-4 \in L^{(4)}$.

Let $D_{4}(x, a)+b$ be reducible. Then, according to $2.2(\mathrm{a}), D_{2}\left(x, a^{2}\right)+b$ is reducible or $\beta \in-4 L^{(4)}$. Thus $2 a^{2}-b \in K^{(2)}$ or $\beta \in L^{(4)}$. Conversely, suppose that $2 a^{2}-b \in K^{(2)}$ or $\beta \in L^{(4)}$. If $2 a^{2}-b \in K^{(2)}$ then $D_{2}\left(x, a^{2}\right)+b$ is reducible and hence $D_{4}(x, a)+b$ is reducible (by $1.3(\mathrm{a})$ ). Note that $b^{2}-4 a^{4} \notin K^{(2)}$ since $\beta \notin K$. Hence if $2 a^{2}-b \notin K^{(2)}$ then $-2 a^{2}-b \in K^{(2)}$, and then 2.2(b) implies that $D_{4}(x, a)+b$ is reducible since $\beta \in-4 L^{(4)}$.

3.5 Lemma. Let $|K|=q<\infty$ and let e denote the order of $a \in K^{\times}$.

(a) $a \in K^{(m)}$ iff $(q-1, m) \mid(q-1) / e$.

(b) Let $p$ be a prime. Then $a \notin K^{(p)}$ iff $p \mid e$ and $p \nmid(q-1) / e$.

(c) Let $a \notin K^{(2)}$. Then $a \notin-4 K^{(4)}$ iff $4 \mid q-1$.

Proof. Part (a) is a simple consequence of the fact that $K^{\times}$is a cyclic group of order $q-1$. Assertion (b) follows from (a) (since $e \mid q-1$ ). Now let $a \notin K^{(2)}$. Note that $q$ is odd. If $4 \nmid q-1$ then $-1 \notin K^{(2)}$ and hence $-a / 4 \in K^{(2)}$. From $(4, q-1)=2$ we obtain $K^{(2)}=K^{(4)}$ and hence $-a / 4 \in K^{(4)}$. Conversely, $-a / 4 \in K^{(4)}$ implies that $-1 \notin K^{(2)}$ and hence $4 \backslash \chi q-1$. Thus (c) is proved.

The following result is, in a weaker form, due to Gao and Mullen [2]. (Remark 3.7 shows that their result follows from 3.6. In [2] it is also required that conditions similar to those given below hold for every $e$ which is the order of a zero of $x^{2}+$ $b x+a^{n}$.)

3.6 Theorem. Let $|K|=q<\infty$ and $n>2$. Let $a \in K^{\times}$and $b \in K$. Let e denote the order $\left(\right.$ in $\left.\bar{K}^{\times}\right)$of some zero of $x^{2}+b x+a^{n}$. Then $D_{n}(x, a)+b$ is irreducible iff the following conditions hold:

(i) Every odd prime factor of $n$ divides e but does not divide $\left(q^{2}-1\right) / e$.

(ii) If $n$ is even and $e \mid q-1$ then $b^{2} \neq 4 a^{n}, 2 \mid e, 2 e \nmid q-1$, and if $4 \mid n$ then $4 \mid q-1$.

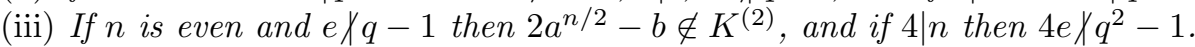


Proof. If $e \mid q-1$ then for every odd prime $p \mid e$ we have $(p, q+1)=1$. Hence in (i) we may replace $\left(q^{2}-1\right) / e$ by $(q-1) / e$ if $e \mid q-1$. Let $\beta \in \bar{K}$ be a zero of $x^{2}+b x+a^{n}$ with order $e$. Note that $\beta \in K$ iff $e \mid q-1$. Let $L=K(\beta)$.

First we suppose that $n$ is odd. Then 2.1(a) shows that $D_{n}(x, a)+b$ is irreducible iff $x^{n}-\beta$ is irreducible over $L$. The latter condition holds (by 2.3) iff $\beta \notin L^{(p)}$ for every prime $p \mid n$. The assertion thus follows from 3.5(b).

Let $n=2 m$. If $e \mid q-1$ then the assertion follows from 2.1(b) by making use of 3.1 and 3.5. In the sequel we assume that $e \bigwedge q-1$. Note that $D_{2}\left(x, a^{m}\right)+b$ is irreducible iff $2 a^{m}-b \notin K^{(2)}$. If $4 \not \backslash n$ then the assertion follows from 2.4 by making use of what we have already proved. If $4 \mid n$ then, in addition, we employ 3.4. The assertion follows since $2 a^{m}-b \notin K^{(2)}$ implies that $q$ is odd and hence $\beta \in L^{(4)}$ holds iff $e \mid\left(q^{2}-1\right) / 4$ (by 3.5(a)).

3.7 Remark. We have $e \mid q^{2}-1$. If $e \mid q-1$ then $b^{2}-4 a^{n} \in K^{(2)}$. If $e \nmid q-1$ then $b^{2}-4 a^{n} \notin K^{(2)}$, or $q$ is even and $b \neq 0$.

If (ii) or (iii) holds then $q$ is odd. If (iii) holds then $b^{2}-4 a^{n} \notin K^{(2)}$ and $2 a^{n / 2}-b \notin K^{(2)}$. Then it follows that $-2 a^{n / 2}-b=c^{2}$ with $c \in K^{\times}$and hence from $\beta^{2}+b \beta+a^{n}=0$ we obtain $\beta=\left(\beta-a^{n / 2}\right)^{2} / c^{2} \in L^{(2)}$. Thus (iii) implies that $2 e \mid q^{2}-1$; and if $4 \mid n$ then it follows that $2 \mid e\left(\right.$ since $\left.4 \mid q^{2}-1\right)$.

\section{REFERENCES}

[1] W.-S. Chou: The factorization of Dickson polynomials over finite fields, Finite Fields Appl. 3 (1997), 84-96. CMP 97:07

[2] S. Gao and G. L. Mullen: Dickson polynomials and irreducible polynomials over finite fields, J. Number Theory 49 (1994), 118-132. MR 95i:11143

[3] S. Lang: Algebra (Third Edition), Addison-Wesley, Reading, 1993.

[4] R. Lidl, G. L. Mullen, and G. Turnwald: Dickson Polynomials, Pitman Monographs and Surveys in Pure and Applied Mathematics 65, Longman, Essex, 1993. MR 94i:11097

[5] L. Rédei: Algebra, Geest \& Portig, Leipzig, 1959. (Pergamon Press, London, 1967.) MR 21:4885; MR 35:2697

[6] T.J. Rivlin: Chebyshev Polynomials (Second Edition), Wiley, New York, 1990. MR 92a: 41016

[7] A. Schinzel: Selected Topics on Polynomials, University of Michigan Press, Ann Arbor, 1982. MR 84k: 12010

Mathematisches Institut, Universität Tübingen, Auf der Morgenstelle 10, D-72076 Tübingen, Germany

E-mail address: gerhard.turnwald@uni-tuebingen.de 